infiltration with DW-MRI had a sensitivity, specificity and accuracy of $76.2 \%, 84.4 \%$ and $82.9 \%$ respectively. Association of both techniques increased sensitivity and specificity up to $84.6 \%$ and $93.2 \%$ and provided a low false negative rate $(2.3 \%)$.

Conclusion* The combination of 3D-TVUS and DW-RMI offers a high sensitivity and specificity to identify deep myometrial infiltration in patients with endometrioid G1 or G2 EC, thus these patients might benefit from performance of both techniques in preoperative evaluation.

\section{THE SIGNIFICANCE OF SINGLE VERSUS MULTIPLE POLYPS AT HYSTEROSCOPY IN POST MENOPAUSAL BLEEDING}

J Davies, L Honeyman, K Hartshorn, J Dasgupta, A Phillips, S Kolhe. Derby Gynaecological Cancer Centre, Derby, UK

\subsection{6/ijgc-2021-ESGO.102}

Introduction/Background* The significance of endometrial polyps in women presenting with post menopausal bleeding (PMB) is uncertain, with variable risk of malignancy between $3.5 \%$ and $6 \%$. Outpatient hysteroscopy is now the standard of care for endometrial assessment with hysteroscopic polypectomy is increasingly performed in this setting. We aimed to establish the relevance and malignancy risk in women with solitary or multiple polyps presenting with PMB.

Methodology A retrospective review of prospectively recorded data between October 2017 and December 2019; during which 449 patients underwent outpatient hysteroscopy and polypectomy for bleeding. Records were interrogated for patient, procedural and histological factors.

Result(s)* The mean age of patients undergoing polypectomy was 63 [IQR 55-70] years. Of these only 69/449 (15\%) did not have polyps detected on ultrasound prior to hysteroscopy. Vaginoscopic hysteroscopy was attempted in 398 cases was successful in 359 (90\%) of cases. Quality of assessment was determined by stated visualization of both ostia, this was achieved in $400(89 \%)$ cases. Only 98 cases (21\%) were described as difficult of which the main causes were cervical stenosis, cervical tortuosity or uterine lie.

For patients undergoing a single polypectomy $(n=286) .276$ cases were benign, 9 had hyperplasia without atypia, 7 had hyperplasia with atypia and 17 had cancers with one sample insufficient.

For patients undergoing multiple polypectomy $(n=162)$. 137 cases were benign, 10 had hyperplasia without atypia, 7 had hyperplasia with atypia and cancer was diagnosed in 21 .

The rate of cancer in multiple polyps and single polyps was $13 \%$ and $6 \%$ respectively, with multiple polyps conveying a $2.04 \mathrm{x}$ increased risk of malignancy.

Conclusion* Outpatient polypectomy is a safe and well tolerated procedure with low complication rates. Multiple polyps can be resected in outpatient see-and-treat setting and should be encouraged due to the increased rates of cancer seen in those with multiple polyps.

\section{TRANSVAGINAL ULTRASOUND-GUIDED CORE BIOPSY - OUR EXPERIENCES IN A COMPREHENSIVE CANCER CENTRE}

1;2D Lengyel, 1;2Z Novák, ${ }^{3} \mathrm{~K}$ Kőhalmy, ${ }^{4}$ I Vereczkey. ${ }^{1}$ University of Szeged - Faculty of Medicine, Doctoral School of Clinical Medicine, Szeged, Hungary; ${ }^{2}$ National Institute of Oncology, Department of Gynaecology, Budapest, Hungary; ${ }^{3}$ National Institute of Oncology, Department of Biochemistry, Budapest, Hungary; ${ }^{4}$ National Institute of Oncology, Department of Surgical and Molecular Pathology, Budapest, Hungary

\subsection{6/ijgc-2021-ESGO.103}

Introduction/Background" Histological diagnosis of female pelvic tumours is essential in their adequate and early clinical management. Ultrasound-guided biopsy is a routine diagnostic method to obtain tissue samples and used widely in different types of tumours. Pelvic solid masses can be biopsied via transabdominal, transvaginal, transrectal or transperineal routes. Generally, lesions located deep within the female pelvis are not easy to access transdabdominally due to various bowel loops, major vessels, uterus, urinary bladder and ureter being in the path of the needle.

Methodology We report our experience of transvaginal ultrasound (TVUS)-guided core biopsies involving 303 patients referred to the gynaecological ultrasound unit of our national comprehensive cancer centre. All patients who underwent a transvaginal, ultrasound-guided core needle biopsy sampling between March 2019 and December 2020 were included.

Result(s)* Adequate histologic specimens were obtained in 299 patients (98.7\%). The most common sites of biopsy sampling were the adnexa (29.7\%), the vaginal stump or wall $(13.5 \%)$, the uterus $(11.6 \%)$ and the peritoneum (10.2\%). Malignancy was confirmed in two-thirds of patients (201/303) and a primary malignancy was diagnosed in 111 of the 201 histologically verified malignant cases (55.2\%). Interestingly, 23.9\% (48/201) of malignant tumours were proven to have a non-gynaecological origin. Among them, gastrointestinal tumours occurred the most frequently (31/48 patients). Three abscesses were discovered following the biopsy procedure, resulting in a complication rate of $1 \%$. In $94(31 \%)$ patients, subsequent surgery allowed the comparison of the ultrasound-guided and surgically obtained histologic results. We found inaccuracy in 12 cases (12.8\%), which is discussed in this paper in detail. Sensitivity, specificity, PPV and NPV to diagnose malignancy was $94.8 \%$, 94.1\%, 98.7\% and 80.0\%, respectively.

Conclusion* According to our experience, TVUS-guided NCB is a safe and effective histological sampling procedure, providing adequate tissue for pathological evaluation in $99 \%$ of cases. It can reliably guide therapy as its performance is satisfactory compared to surgically obtained histology. As infectious complications might rarely occur, routine preoperative vaginal disinfection is suggested. In case of the suspicion of malignancy despite negative biopsy histology, further investigation is proposed due to the $80 \%$ NPV.

\section{BONE SCAN IN GYNECOLOGICAL CANCER}

CF Ghomari*, A Medjahedi. Nuclear Medicine, Tlemcen University Hospital. Dr Tidjani Damerdji; Tlemcen University, Medicine Departement; Tlemcen, Algeria

10.1136/ijgc-2021-ESG0.104

Introduction/Background* Bone metastases of gynecological cancer are rare. To complete the initial staging of the disease, 
a combination of BS (bone scan) and CT (computerized tomography scan) is essential considering the unavailability of PET scan (positron emission tomography).

The aim of this study is to explore the bone involvement of gynecological cancer.

Methodology It is a retrospective study including gynecological cancer patients, referred for bone scan during 2019, at the Nuclear Medicine department of Tlemcen University Hospital in Algeria.

Two hours after the intravenous injection of $8-10 \mathrm{MBq} / \mathrm{kg}$ ${ }^{99 \mathrm{~m}}$ Tc-HMDP (hydroxy methylene diphosphonate), whole body scanning is accomplished by dual head hybrid gamma camera with low energy high resolution collimator.

Result(s)* Twenty-three bone scans were indicated in the initial staging of gynecological cancer patients, median age of 59 years [42-81], to explore bone pain in 15 cases $(65 \%)$ or an advanced stage of the neoplasms (peritoneal carcinomatosis, bone metastases or lung metastases) in 08 cases (35\%).

The different types of tumors are ovarian $(10 ; 43,5 \%)$, cervical $(9 ; 39,1 \%)$, and endometrial $(4 ; 17,4 \%)$.

The majority of BS (15 patients; 65,2\%) are relevant of osteoarthritis, involving both the spine and the peripheral joints, which are symptomatic in knees and shoulders. 4 patients $(17,4 \%)$ had normal scintigraphy.

Four patients $(17,4 \%)$ had multiple bone metastases with hot uptake scintigraphic pattern, involving both the axial and appendicular skeleton. Among these cases, the most common metastatic sites are pelvis, spine, and rib. Upon these metastatic patterns, 2 cases are related to endometrial cancer $(8,7 \%)$, the rest are related to cervical $(1 ; 4,34 \%)$ and ovarian carcinoma $(1 ; 4,34 \%)$. Bone pain was present in 3 metastatic cases involving spine, the rest was declared with bone metastases at CT.

Conclusion* Bone involvement of gynecological cancer is not frequent, it can be symptomatic or reported by CT scan. BS is a useful tool to explore with its good sensitivity the entire skeleton, in order to stage the neoplasm.

\section{PAP-SMEARS ALLOW THE IDENTIFICATION OF PROTEIN BIOMARKERS TO DIAGNOSE ENDOMETRIAL CANCER}

${ }^{1} S$ Cabrera*, ${ }^{2} E$ Coll-de la Rubia, ${ }^{3} E$ Martínez Garcia, ${ }^{3} \mathrm{~A}$ Lesur, ${ }^{4} \mathrm{~A}$ Reques, ${ }^{5} \mathrm{MA}$ Casares de Cal, ${ }^{5} \mathrm{~A}$ Gomez Tato, ${ }^{6} \mathrm{E}$ Sabidó, ${ }^{6} \mathrm{E}$ Borrás, ${ }^{7} \mathrm{R}$ Peiró, ${ }^{3} \mathrm{G}$ Dittmar, ${ }^{1} \mathrm{~A}$ Gil-Moreno, ${ }^{2} \mathrm{E}$ Colas. ${ }^{\prime} G y n e c o l o g i c$ Oncology Unit, University Hospital Vall d'Hebron, Vall d'Hebron Barcelona Hospital Campus and Autonomous University of Barcelona (UAB); ${ }^{2}$ Biomedical Research Group in Gynecology, Vall Hebron Institute of Research, Universitat Autònoma de Barcelona, CIBERONC, 08035 Barcelona, Spain; ${ }^{3}$ Quantitative Biology Unit, Luxembourg Institute of Health, L-1445 Strassen, Luxembourg; ${ }^{4}$ Pathology Department, Vall Hebron University Hospital, CIBERONC, 08035 Barcelona, Spain; ${ }^{5}$ University of Santiago de Compostela, Faculty of Mathematics, Santiago de Compostela, Spain; ${ }^{6}$ Center for Genomic Regulation, Proteomics, Barcelona, Spain; ${ }^{7}$ Hospital General de Catalunya, Sant Cugat del Vallés, Spain

\subsection{6/ijgc-2021-ESGO.105}

Introduction/Background* Endometrial cancer (EC) is the most common gynecological cancer in developed countries. There are no screening tools for its early diagnosis, and the diagnostic process starts with the apparition of related symptoms, mainly, abnormal vaginal bleeding (AVB). It is estimated that $\sim 7 \mathrm{M}$ women with AVB will undergo the diagnostic process every year in Europe, and from those, 9\% will have EC. Importantly, the current diagnostic process relies on the pathological examination of an endometrial biopsy that is always obtained by minimally-invasive to invasive methods. This overdiagnosis creates a big burden to the healthcare systems, so the development of non-invasive tools for EC diagnosis would revolutionize this scenario. Our aim is to approach a non-invasive diagnosis by the identification of protein biomarkers to accurately diagnose EC in liquid cervical cytologies.

Methodology The discovery phase consisted of a shotgun label-free proteomic approach. It included 60 patients $(20 \mathrm{EC}$, 20 controls suffering AVB without endometrial or cervical pathology, and 20 controls without endometrial pathology but cervical pathology). The levels of a statistically significant set of 75 proteins (110 peptides) from the discovery phase were measured in a verification phase including 234 (107 non-EC; 127 EC) patients by LC-MSMS/PRM. Analysis was performed using MaxQuant, Skyline, SPSS and R software.

Result(s)* The discovery study permitted to determine a total number of 2,888 proteins in our samples. Statistical analysis identified 75 potential proteins differently expressed between EC and non-EC patients to be further assessed and verified. Verification phase revealed the potential of 58 proteins measured in cervical cytologies to reach a non-invasive diagnosis of EC. Specifically, 16 proteins achieved an AUC $>0.75$, and 3 proteins an AUC $>0.8$. Additionally, an ELISA assay of the best performing protein was tested reproducing the results obtained by mass-spectrometry and reaching an $\mathrm{AUC}=0.927$ in this dataset.

Conclusion* We identified protein biomarkers in liquid cervical cytologies to diagnose EC patients with a diagnostic power up to $92 \%$. These results are promising for a subsequent development of an early and non-invasive screening tool for EC. This tool is expected to change the standard of care in EC diagnosis.

\section{7 EFFICACY OF TRANSVAGINAL AND TRANSABDOMINAL ULTRASOUND GUIDED BIOPSIES FOR HISTOLOGICAL DIAGNOSIS AND COMPLICATION RATES IN THE TWO BIOPSY METHODS}

B Hamid*, G Dadayal. Airedale General Hospital, Radiology department, Steeton, UK

\subsection{6/ijgc-2021-ESG0.106}

Introduction/Background* Thousands of women each year of varying age undergo Transvaginal (TV) and Transabdominal (TA) guided biopsy's due to suspected gynaecological cancers, the most common being Ovarian Cancer \& Primary Peritoneal Carcinomatosis. This study firstly looked at the efficiency and accuracy in attaining a conclusive histological diagnosis from a biopsy obtained via TA and TV method.

It also looked at whether there was any correlation between complications of TA and TV guided biopsies, and if so, were any confounding factors identified.

Methodology A search was done to identify all individuals who had undergone an ultrasound guided biopsy at Airedale General Hospital between March 2018 and August 2020. Those individuals who had undergone ultrasound guided biopsies at sites other than TA and TV were excluded from this study (e.g. axillary). 